\title{
J Wave Autodetection Using Analytic Time-Frequency Flexible Wavelet Transformation Applied on ECG Signals
}

\author{
Deng-ao Li $\mathbb{D}^{D}$, Jie Zhou $\left(\mathbb{D}\right.$, Jumin Zhao ${ }^{(D)}$, and Xinyan Liu \\ College of Information and Computer, Taiyuan University of Technology, Taiyuan, China \\ Correspondence should be addressed to Deng-ao Li; lidengao@tyut.edu.cn
}

Received 18 November 2017; Revised 16 March 2018; Accepted 12 April 2018; Published 31 May 2018

Academic Editor: Li Xu

Copyright (c) 2018 Deng-ao Li et al. This is an open access article distributed under the Creative Commons Attribution License, which permits unrestricted use, distribution, and reproduction in any medium, provided the original work is properly cited.

\begin{abstract}
As a new important index of the electrocardiogram (ECG) of ventricular bipolar play, J wave plays an increasingly significant role in the clinical diagnosis. The existence of J wave hints at potential crisis of fatal disease and even death. Nowadays, however, it can hardly meet the clinical needs where the diagnosis of J wave variation only depends on experience of clinicians. Therefore, a new technique which is capable of detecting J wave using analytic time-frequency flexible wavelet transformation (ATFFWT) is proposed in this paper. We have used ATFFWT to decompose the processed ECG signals into the desired subbands. Further, Fuzzy Entropy (FE) is computed from each subband to capture more hidden and meaningful information. Feature scoring method is applied to select optimal feature set. Finally, the extracted features are fed to Least Squares-Support Vector Machine (LS-SVM) classifier. The 10-fold cross validation is used to obtain reliable and stable performance and to avoid the overfitting of the model. Our proposed algorithm has achieved accuracy of 97.61\% for Morlet Wavelet (MW) kernel in comparison to 97.56\% for Radial Basis Function (RBF) kernel. The developed effective algorithm can be used to design an expert system to aid clinicians in their regular diagnosis.
\end{abstract}

\section{Introduction}

Nowadays, cardiovascular diseases (CVDs) cause nearly onethird of all deaths worldwide. CVDs remain a leading cause of health loss for all regions of the world and a major barrier to long-term sustainable development of mankind [1]. Nearly 17 million people die due to cardiovascular diseases globally every year [2]. J wave is regarded as a new important index of the electrocardiogram (ECG) of ventricular bipolar play, and it plays an increasingly significant role in the clinical diagnosis of cardiovascular diseases. A series of diseases or conditions that can produce J waves in the ECG continues to rise [3].

The J wave, also referred to as an Osborn wave, is a deflection immediately following the QRS complex of the surface ECG, which is usually partially buried inside the QRS, often appearing as a J-point elevation; it represents the end of depolarization and the start of bipolarization [4]. The presence of J wave may lead to early repolarization syndrome (ERS), pericarditis, idiopathic ventricular fibrillation (IVF),
Brugada syndrome (BrS), and even sudden unexplained nocturnal death syndrome [4]. From a mechanistic point of view, these syndromes should be referred to as the J wave syndromes [4]. J wave and J wave syndrome are high-risk early warning indicators of sudden cardiac death [5]. The appearances of prominent $J$ waves in the ECG have long been reported in cases of hypothermia and hypercalcemia [6]. More recently, accentuation of the J wave has been associated with life-threatening ventricular arrhythmias. Although typical J waves usually are accentuated with bradycardia or long pauses, the opposite has also been described $[5,7]$. J waves are often seen in young males with no apparent structural heart diseases, whereas intraventricular conduction delay is often observed in older individuals or those with a history of myocardial infarction or cardiomyopathy $[5,7]$.

J wave is mixed in the normal ST segment, coupled with the small amplitude, existence of noise, and baseline wander. Accordingly, the diagnosis of J wave variation and minute changes in the ECG signals only depends on the clinicians' experience, which can not meet demand at present 


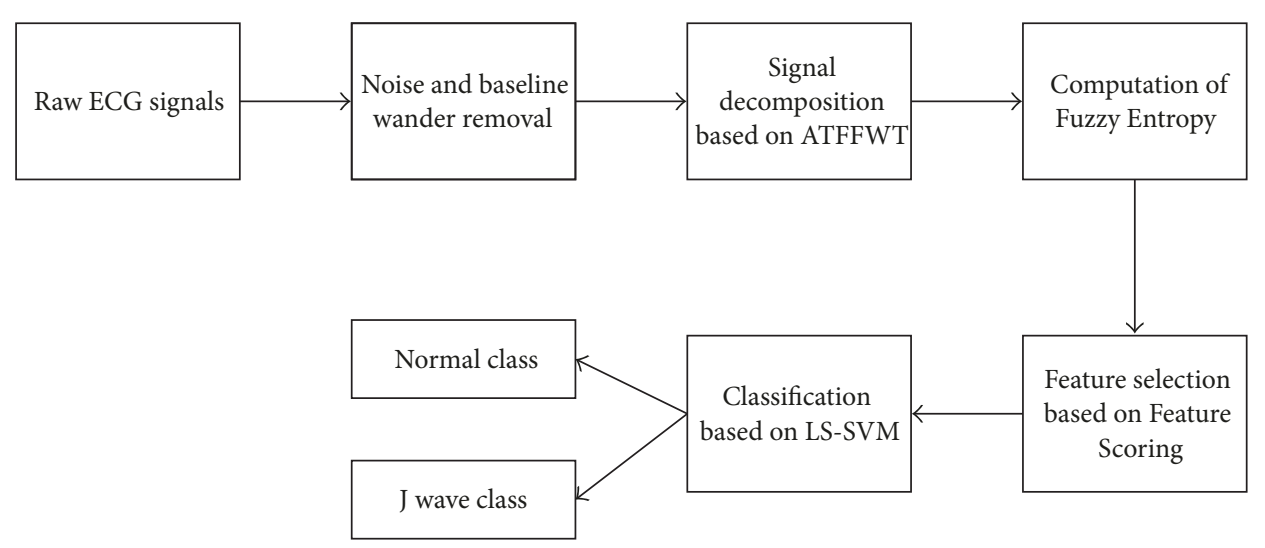

FIGURE 1: Steps used for automatic J wave detection.

clinical, and is also apt to be misdiagnosed. Therefore, it is pretty essential and necessary for us to analyze J wave from the perspective of computer aided method with advanced digital signal processing techniques and machine learning algorithms, which can help to capture the subtle and hidden information in the ECG signals and realize the accurate and automatic diagnosis of J wave. Such an automated system will provide tremendous assistance to the clinicians in their routine screening of cardiac patients [8-12]. In literature, although the method of processing ECG signals has been very mature, methods of detecting J wave signals from ECG signal were relatively less and the detection precision is undesirable. The authors in [13] proposed the method based on locating a break point in the descending limb of the terminal QRS. They added new logic to Glasgow ECG analysis program to automate the detection of $\mathrm{J}$ wave. A technique based on digital 12-lead electrocardiogram is used in [14]. ECG signals were automatically processed with the GE Marquette 12SL program 2001 version (GE Marquette, Milwaukee, WI). And the functional data analysis techniques were applied to the processed ECG signals. In [15], five features including three time-domain features and two wavelet-based features are defined; these features are found significantly different in discriminate J wave and normal classes. Thereafter, Principle Component Analysis (PCA) is used to reduce the dimension of these features. An approach for J wave autodetection based on SVM is proposed. Curving Fitting (CF) and wavelet transform (WT) are used for feature extraction from J wave and healthy ECG segments data in [16], which have shown effective variations for J wave and normal subjects. In [17], a novel $\mathrm{J}$ wave detection method based on massive ECG data and MapReduce is presented. The power spectrum and the cumulative probability of ECG signals are computed as features and Decision Tree (DT) is applied for classification.

Compared with the existing methods of J wave detection, the main contributions of this work are listed as follows: (1) we built a J wave detection expert system with FE entropy on the coefficient of ATFFWT as feature and LS-SVM as a classifier, and it owned high accurate rate; (2) we use lower number of features because feature scoring method is applied (only entropy features) to obtain the desirable accuracy; the detection of J wave will be fast; (3) it is the first time that tenfold cross validation method is applied, which makes our algorithm more reliable and robust. The objective of present work is to develop a noninvasion marker for some cardiac diseases clinically that can automatically, accurately, and fast detect J wave. To achieve this, the ECG signals are segmented into beats firstly. Then, to cope with the nonlinear and nonstationary nature of ECG signals, analytic time-frequency flexible wavelet transformation (ATFFWT) is employed to decompose the signals in terms of subbands signals. Further, Fuzzy Entropy (FE) is computed on decomposed subband. Then, feature scoring method is performed on these FE to select more meaningful features and improve the classification performance. Finally, these clinically significant features are fed to Least Squares-Support Vector Machine (LS-SVM) classifier with different kernel functions. The followed steps of the proposed method are shown in Figure 1.

\section{Materials and Methods}

2.1. Data Acquisition and Preprocessing. In this work, the normal ECG signals (normal class) were recorded from 58 normal subjects, which are acquired from MIT/BIH Normal Sinus Rhythm (NSR) database [12] and Fantasia [12, 18]. From the MIT/BIH NSR dataset, we have used 18 subjects ( 5 males and 13 females). From the Fantasia dataset, we have obtained the ECG records of 40 subjects (20 young and 20 old). The ECG signals with J wave (abnormal class) of 15 patients (10 males and 5 females) are obtained from Shanxi Dayi Hospital; the sampling frequency is $257 \mathrm{~Hz}$. The age of all the subjects varies from 20 to 71 years. ECG signals of lead II were applied in our study. All ECG signals we used were initially inspected by experienced cardiologists. To avoid the inclusion of noise, artifacts, and baseline wander, the digitized ECG signals were preprocessed by using eight levels' Daubechies 6 (db6) basis function of wavelet [8].

2.2. Beats Segmentation. In order to segment the ECG signals into single beats, R-points should be detected firstly. In the 
TABLE 1: Total number of beats used in this work.

\begin{tabular}{lc}
\hline Type & Number of beats \\
\hline J wave & 25,468 \\
Normal & 169,426 \\
Total & 194,894 \\
\hline
\end{tabular}

present study, Pan-Tompkins algorithm is carried out on each preprocessed ECG signal to detect R-peaks [8, 19]. Then ECG beats are segmented or selected through the detected R-points [20]. We chose 64 samples before the R-point and 105 samples after R-point for each ECG beat. Hence, there is a segment of 170 samples for one ECG beat, and it consists of P, QRS, T, and U waves. The number of ECG beats segmented for $J$ wave and normal in this work are shown in Table 1.

2.3. Decomposition of the Beats Based on ATFFWT. Wavelet transform (WT) is a powerful mathematical tool for processing nonstationary signal [10]. WT and its various improved methods are still playing a significant role in the signal processing field, because it enjoys fine time-frequency concentration and multiresolution analysis property. However, WT and some of its improved methods are suffering from a number of limitations and shortcomings. For instance, the continuous WT (CWT) suffers restricted computational efficiency, the discrete WT (DWT) suffers from the limitation of having constant time-frequency covering at all scales, and it also suffers shift-variance and poor resolution at its high frequency subbands [21]. These limitations are addressed by a newly introduced time-frequency analysis tool called ATFFWT [11], which has desirable properties such as shiftinvariance, flexible time-frequency covering, and tunable oscillatory bases [11]. It has been applied to the weak fault features detection in rotating machinery [21] and the diagnosis of coronary artery disease using HRV signals [9]. ATFFWT is realized by the iterated filter bank (IFB) which consists of one low-pass channel and two high-pass channels; one of these high-pass channels analyzes "positive frequencies," while the other analyzes "negative frequencies" [11]. Jth level decomposition of ATFFWT can be achieved by using IFB [11].

Frequency response of the low-pass filter can be given by the following mathematical equations [11]:

$$
\begin{aligned}
& H(w) \\
& = \begin{cases}\sqrt{p q}, & |w|<w_{p} \\
\sqrt{p q} \theta\left(\frac{w-w_{p}}{w_{s}-w_{p}}\right), & w_{p} \leq w \leq w_{s} \\
\sqrt{p q} \theta\left(\frac{\pi-\left(w-w_{p}\right)}{w_{s}-w_{p}}\right), & -w_{s} \leq w \leq-w_{p} \\
0, & |w|>w_{s},\end{cases}
\end{aligned}
$$

where $p$ and $q$ are up and down sampling parameters for lowpass filter, respectively. $w_{s}$ and $w_{p}$ represent the stop band and pass band frequencies of the low-pass filter and are shown as [11]

$$
\begin{aligned}
& w_{p}=\frac{(1-\beta) \pi}{p}+\frac{\varepsilon}{p}, \\
& w_{s}=\frac{\pi}{q} .
\end{aligned}
$$

The other used filter is the high-pass filter and can be defined mathematically as [11]

$$
\begin{aligned}
& G(w) \\
& = \begin{cases}\sqrt{r s} \theta\left(\frac{\pi-\left(w+w_{0}\right)}{w_{1}-w_{0}}\right), & w_{0}<w<w_{1} \\
\sqrt{r s}, & w_{1} \leq w \leq w_{2} \\
\sqrt{r s} \theta\left(\frac{w-w_{2}}{w_{3}-w_{2}}\right), & w_{2} \leq w \leq w_{3} \\
0, & w \in\left[0, w_{0}\right) \cup\left(w_{2}, 2 \pi\right),\end{cases}
\end{aligned}
$$

where $r$ and $s$ are up and down sampling parameters for highpass filter, respectively. The other parameters are described as follows [11]:

$$
\begin{aligned}
w_{0} & =\frac{(1-\beta) \pi}{r}+\frac{\varepsilon}{r}, \\
w_{1} & =\frac{p \pi}{q r} \\
w_{2} & =\frac{\pi-\varepsilon}{r}, \\
w_{3} & =\frac{\pi+\varepsilon}{r}, \\
\varepsilon & \leq\left(\frac{p-q+\beta q}{p+q}\right) \pi .
\end{aligned}
$$

In this work, the transition band $\theta(\omega)$ is chosen as [11]

$$
\theta(\omega)=\frac{[1+\cos (\omega)] \sqrt{2-\cos (\omega)}}{2} \quad \omega \in[0, \pi] .
$$

The parameters $p, q, r, s$, and $\beta$ provide flexibility to control wavelets with the attractive quality-factor ( $Q$-factor) $Q$, dilation factor $d$, and redundancy factor $R$. $\beta$ and $\varepsilon$ are the nonnegative constants. These parameters are not independent of each other and are given as [11]

$$
\begin{aligned}
& Q \approx \frac{2-\beta}{\beta}, \\
& d \approx \frac{p}{q}, \\
& \beta \leq 1, \\
& R \approx\left(\frac{r}{s}\right) \frac{1}{1-d}, \\
& R>\frac{\beta}{1-d} .
\end{aligned}
$$


The perfect reconstruction filter bank can be achieved by satisfying the following condition [11]:

$$
\begin{aligned}
|\theta(w)|^{2}+|\theta(\pi-w)|^{2} & =1, \\
1-\frac{p}{q} & \leq \beta \leq \frac{r}{s} .
\end{aligned}
$$

Hilbert transform pairs of the wavelet bases can be obtained owing to this type of separation of positive and negative frequencies. These characters make ATFFWT flexible by allowing one to control the Q-factor, redundancy, and dilation factor [11]. Recently, it has been applied for characterization of coronary artery disease [22, 23], myocardial infarction ECG signals [23], and detection of congestive heart failure using heart rate variability (HRV) signals [24]. Matlab toolbox of ATFFWT method is available at http://web.itu.edu.tr/ibayram/AnDWT/.

2.4. Nonlinear Feature Extraction from the Detail Coefficients. Fuzzy Entropy is extracted as nonlinear feature from the each beat segment from the standard ECG signals. Therefore, Fuzzy Entropy is computed on the real value of detail coefficients at each level. As an improvement of the sample entropy algorithm, the similarity measures are fuzzed by Fuzzy Entropy (FE) using an exponential function. The steps for the calculation of the FE are as follows [25].

(1) The sampling sequences of length $N$ are extracted from the detail coefficients:

$$
\{u(i): 1 \leq i \leq N\} .
$$

(2) A set of $m$-dimensional vectors refactored and generated in sequential order $X_{i}^{m}=\{u(i), u(i+1), \ldots, u(i+m-$ 1) $\}-u^{0}(i)(i=1, \ldots, N-m) \cdot u^{0}(i)$ represents the mean value and can be defined as [25]

$$
u^{0}(i)=\frac{1}{m} \sum_{j=0}^{m-1} u(i+j)
$$

(3) The distance $d_{i j}^{m}$ between the sequences $X_{i}^{m}$ and $X_{j}^{m}$ is defined as the largest difference, and it can be expressed as

$$
\begin{aligned}
d_{i j}^{m} & =d\left[X_{i}^{m}, X_{j}^{m}\right] \\
& =\max _{k \in(0, m-1)}\left\{\left|u(i+k)-u^{0}(i)-u(j+k)-u^{0}(j)\right|\right\} \\
& (i, j \in[1, N-m], j \neq i) .
\end{aligned}
$$

(4) The similarity degree $D_{i j}^{m}$ between the sequences $X_{i}^{m}$ and $X_{j}^{m}$ is computed by applying the fuzzy function as follows [25]:

$$
D_{i j}^{m}=\mu\left(d_{i j}^{m}, n, r\right)=\exp \left(-\frac{\left(d_{i j}^{m}\right)^{n}}{r}\right),
$$

where $\mu, n$, and $r$ stand for the fuzzy function, the gradient, and the width of the exponential function boundary, respectively.
(5) The function $Q^{m}(n, r)$ is defined, and it is shown as follows [25]:

$$
Q^{m}(n, r)=\frac{1}{N-m} \sum_{i=1}^{N-m}\left(\frac{1}{N-m-1} \sum_{j=1, j \neq i}^{N-m} D_{i j}^{m}\right) .
$$

(6) Finally, FE can be computed according to the formula as follows [25]:

$$
\begin{aligned}
\text { Fuzzy En }(m, n, r, N)= & \ln \left[Q^{m}(n, r)\right] \\
& -\ln \left[Q^{m+1}(n, r)\right] .
\end{aligned}
$$

FE more easily identifies the abnormal activities in the signal. Therefore, it is applied to discriminate nonfocal and focal electroencephalogram signals [26], characterize the surface electromyogram signals [25], and diagnose epilepsy [27].

2.5. Features Selection Based on Feature Scoring. Feature selection is widely used in pattern classification and regression to remove the redundant and uncorrelated features in feature space, thus reducing the computational load and improving the classification performance [28]. In this investigation, we employed a feature scoring algorithm to choose the optimal set. This algorithm is also known as mutual information based feature scoring [28]. In this algorithm, score value is calculated for each feature to reflect its usefulness. The larger $S^{q}$, the higher the dependency between the feature values and the class labels. The score value of each feature is evaluated according to the following formula $[29,30]$ :

$$
S^{q}=\sum_{x^{q}} \sum_{y} P\left(x^{q}, y\right) \log \frac{P\left(x^{q}, y\right)}{P(y) P\left(x^{q}\right)} .
$$

The feature matrix $X \in R^{p \times q}$ consists of $p$ number of beat samples and $q$ number of feature attributes. We gave the class label vector $y, y \in R^{p}$ for the feature matrix. The values of $y$ are 1 and -1 corresponding to the class label of normal class and J wave class. The score value of $q$ th feature in the feature vector $x_{p}$ is calculated by using the probability of $q$ th feature $P\left(x^{q}\right)$ and the probability of class level $P(y)$. In this work, the $\mathrm{FE}$ feature vectors and the new feature vectors obtained using feature scoring are fed to LS-SVM.

2.6. Classification Based on LS-SVM. The LS-SVM [31], an excellent binary classifier derived from SVM, has successful application of pattern recognition and nonlinear function fitting. However, some problems exist in SVM such as the parameter selection for hyperplane, and the size of matrix is greatly influenced by the number of training samples in Quadratic Programming (QP) problem solving, resulting in the huge solution dimension. Therefore, the improved method LS-SVM makes up for the limitation of SVM. They minimize the classification error by constructing a hyperplane in higher dimensional space and maximize the 
TABLE 2: $p$ values of features computed from each level of ATFFWT decomposition for J wave and normal classes.

\begin{tabular}{lccccc}
\hline Features & Level 2 & Level 3 & Level 4 & Level 5 & Level 6 \\
\hline FE $p$ value & $8.328 \times 10^{-19}$ & $3.943 \times 10^{-19}$ & $1.791 \times 10^{-19}$ & $1.293 \times 10^{-19}$ & $4.531 \times 10^{-18}$ \\
\hline
\end{tabular}

TABLE 3: Mean $(\mu)$ and standard deviation $(\sigma)$ of features computed from each level of ATFFWT decomposition for J wave and normal classes.

\begin{tabular}{lccc}
\hline Level of decomposition & Features & J wave class $(\mu \pm \sigma)$ & Normal class $(\mu \pm \sigma)$ \\
\hline Level 1 & $\mathrm{FE}_{\mathrm{D} 1}$ & $0.2787 \pm 0.0370$ & $0.2254 \pm 0.1098$ \\
Level 2 & $\mathrm{FE}_{\mathrm{D} 2}$ & $0.2638 \pm 0.2549$ & $0.2396 \pm 0.0723$ \\
Level 3 & $\mathrm{FE}_{\mathrm{D} 3}$ & $0.3081 \pm 0.2995$ & $0.2602 \pm 0.0259$ \\
Level 4 & $\mathrm{FE}_{\mathrm{D} 4}$ & $0.2807 \pm 0.0578$ & $0.2340 \pm 0.1492$ \\
Level 5 & $\mathrm{FE}_{\mathrm{D} 5}$ & $0.1032 \pm 0.0216$ & $0.0984 \pm 0.0743$ \\
\hline
\end{tabular}

distance of any one of the classes. The classification decision function of LS-SVM is defined mathematically as [31]

$$
J=\operatorname{sign}\left[\sum_{m=1}^{M} \alpha_{m} \omega_{m} K\left(z, z_{m}\right)+b\right]
$$

where $K\left(z, z_{m}\right)$ represents a kernel function, $\alpha_{m}$ denotes the Lagrangian multiplier, $z_{m}$ is the $M$ th input vector, $\omega_{m}$ is the target vector, and $b$ represents bias term. In this work, we selected Radial Basis Function (RBF) and Morlet Wavelet (MW) as the kernel function.

The expression of the RBF kernel is given as [32]

$$
K\left(z, z_{m}\right)=e^{-\left\|z-z_{m}\right\|^{2} / 2 \sigma^{2}}
$$

where $\sigma$ is the kernel parameter and it controls the width of the RBF kernel function. MW kernel can be represented as follows $[33,34]$ :

$$
K\left(z, z_{m}\right)=\prod_{n=1}^{D} \cos \left[v_{0} \frac{z^{n}-z_{m}^{n}}{l}\right] e^{-\left\|z^{n}-z_{m}^{n}\right\|^{2} / 2 l^{2}}
$$

where $D$ is the dimension of the feature set and $l$ denotes the scale factor of MW kernel. LS-SVM is widely used in the diagnosis of diabetes [35], analysis of the heart sound signals [36], and classification of focal EEG class [37, 38], seizure class $[26,39]$, and glaucoma using fundus images [40].

\section{Results and Discussion}

In this present study, we have segmented ECG signals into beats. Then, to extract features, the ECG beats are employed to ATFFWT decomposition method to obtain a series of subbands signals. The plots of real values of coefficients are shown in Figures 2(a) and 2(b) for J wave and normal classes, respectively. These subband signals are obtained from ATFFWT-based decomposition of the ECG beats. In Figure 2, $\mathrm{CN}$ is the decomposition detail coefficient and CO stands for the magnitude of the coefficient. Lla and Llb are described as detail coefficients of first level. Similarly, L2a and L1b, L3a and L3b, L4a and L4b, and L5a and L5b represent second-level, third-level, fourth-level, and fifth-level detail coefficients, respectively. The parameters of ATFFWT are selected by way of trial and error experimentation to reach the maximum classification accuracy. In the present study, the values of the parameters are $p=5, q=6, r=1, s=2$, and $\beta=0.8(r / s)[9$, 21]. Therefore, $d=5 / 6, Q=4$, and $R=3$. The high $Q$-factor denotes that desirable frequency analysis of ECG signals is acquired. In order to select the desirable decomposition level, $p$ values from subbands at different levels of ATFFWTbased decomposition are computed using Kruskal-Wallis test [41]. It should be noted that the lowest $p$ value indicates the best discrimination ability of the feature. The lowest $p$ values are observed at the fifth level of decomposition, as can be seen in Table 2. There was no significant improvement when the decomposition is increased from fifth to sixth level. Accordingly, we select fifth level of decomposition for analyzing J wave and normal ECG signals.

FE is computed from detail coefficients, and the parameters demanded to compute FE are also chosen by trial and error experimentation, thus getting maximum classification accuracy; the values of the parameters $m, n$, and $r$ are selected to be 5,2 , and 0.3 separately [27]. Mean $(\mu)$ and standard deviation $(\sigma)$ of the features computed from various levels of ATFFWT decomposition for J wave and normal classes are offered in Table 3, and we can observe from the table that, for $J$ wave classes, $\mu$ and $\sigma$ of the FE features have higher values at each decomposition level as compared to normal classes. Boxplots for FE at various levels of decomposition are depicted in Figures 3(a)-3(e). Feature scoring method is employed for selection, thus removing irrelevant and redundant features, and search for the optimal feature subset. In this work, the dimension of the feature vector is five, and it derives from the FE computation of 5 levels subbands. The score value of each feature evaluated using feature scoring method is shown in Figure 4. It can be observed that $\mathrm{FE}_{\mathrm{D} 1}$, $\mathrm{FE}_{\mathrm{D} 4}$, and $\mathrm{FE}_{\mathrm{D} 5}$ have higher score values than those of $\mathrm{FE}_{\mathrm{D} 2}$ and $\mathrm{FE}_{\mathrm{D} 3}$ features.

Accuracy (ACC), sensitivity (SEN), and specificity (SPE) are computed at each level of decomposition and are tabulated in Table 4. It is obviously seen that the significant improvement in classification performance occurs at second level of decomposition. The average accuracy, average sensitivity, and average specificity of classification are found to be $97.56 \%, 95.69 \%$, and $95.78 \%$ for RBF kernel and $97.61 \%$, $97.76 \%$, and $95.82 \%$ for MW kernel functions, respectively. 

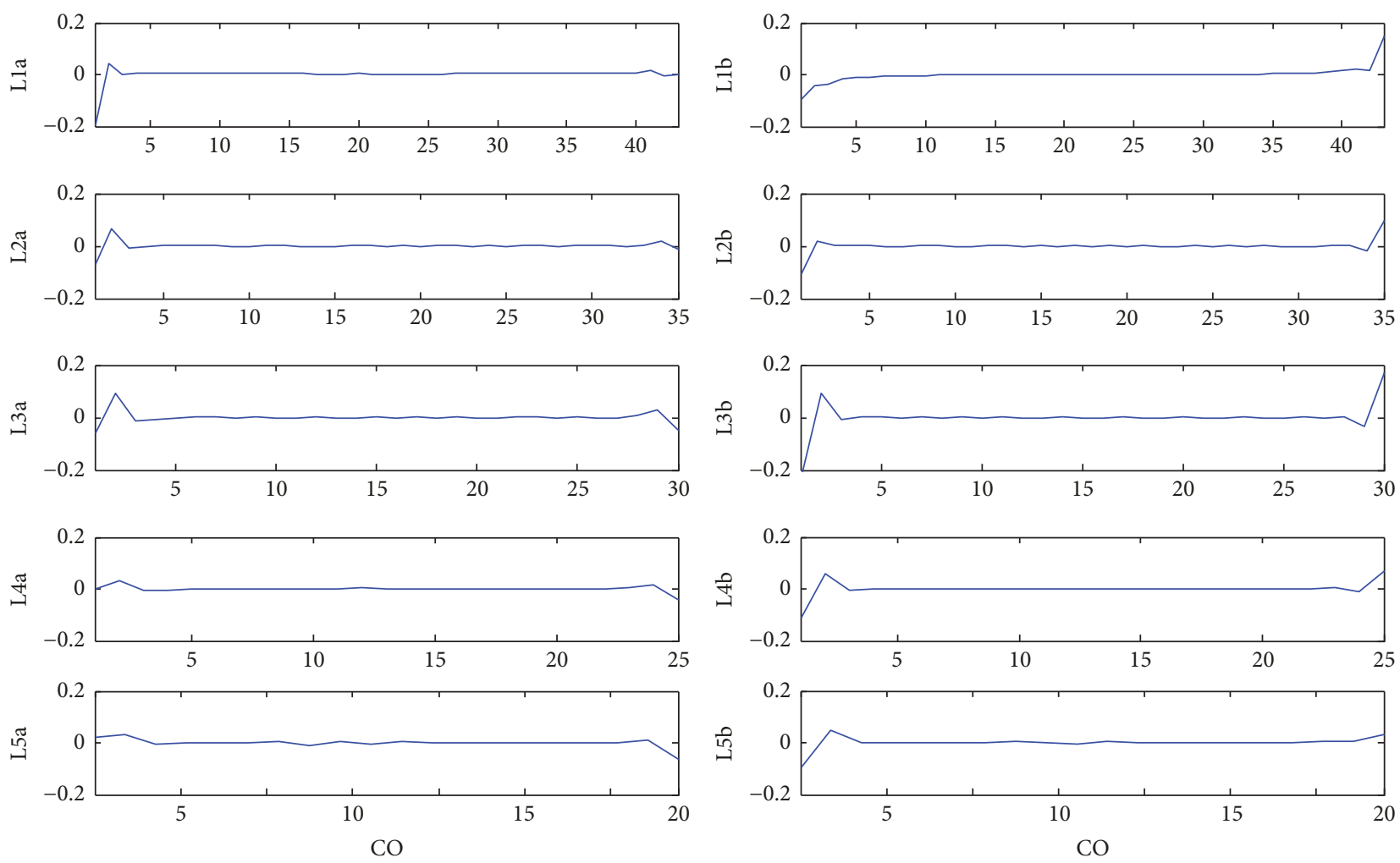

(a)
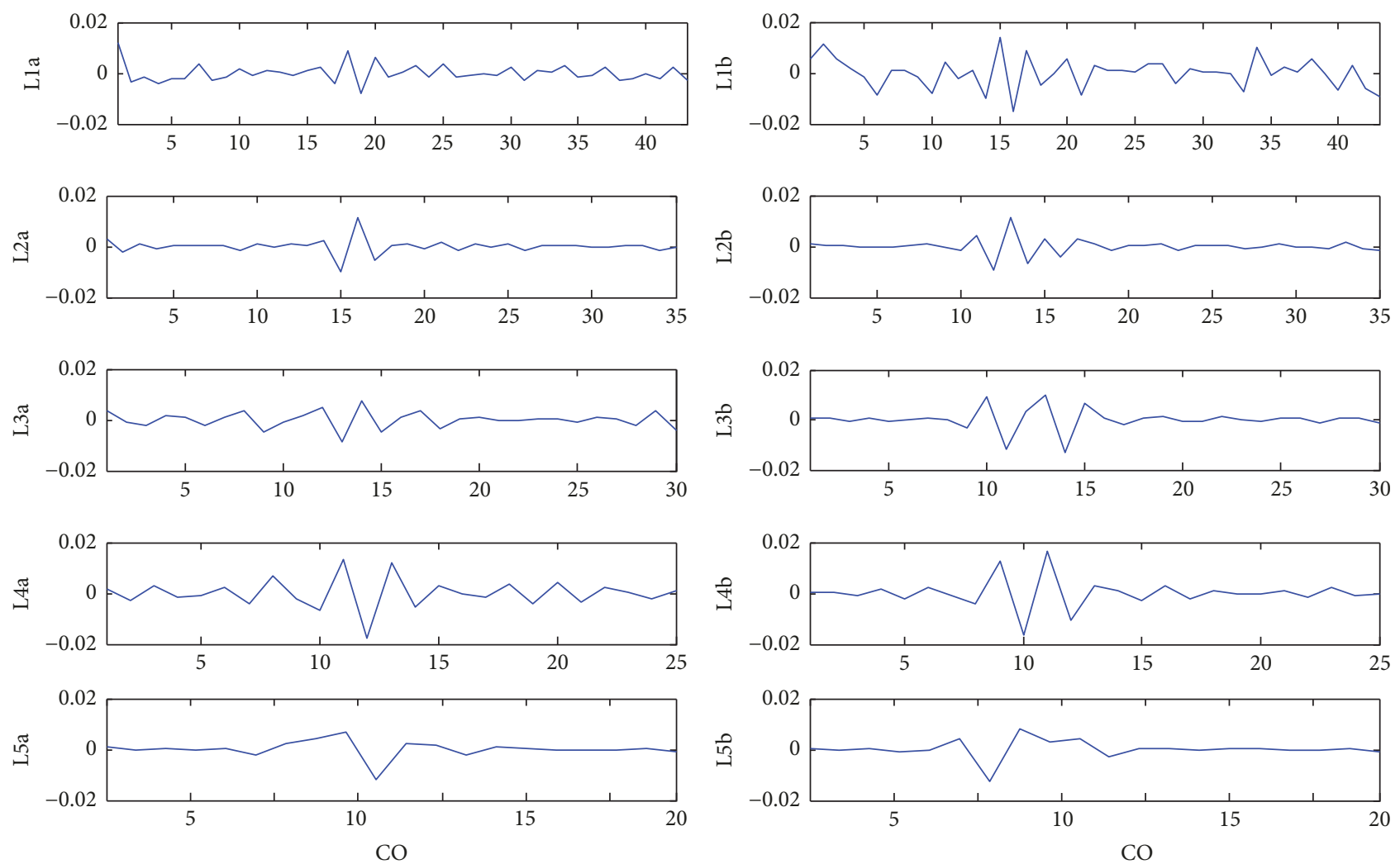

(b)

Figure 2: Plots of real coefficients of ATFFWT decomposition: (a) normal class and (b) J wave class. 


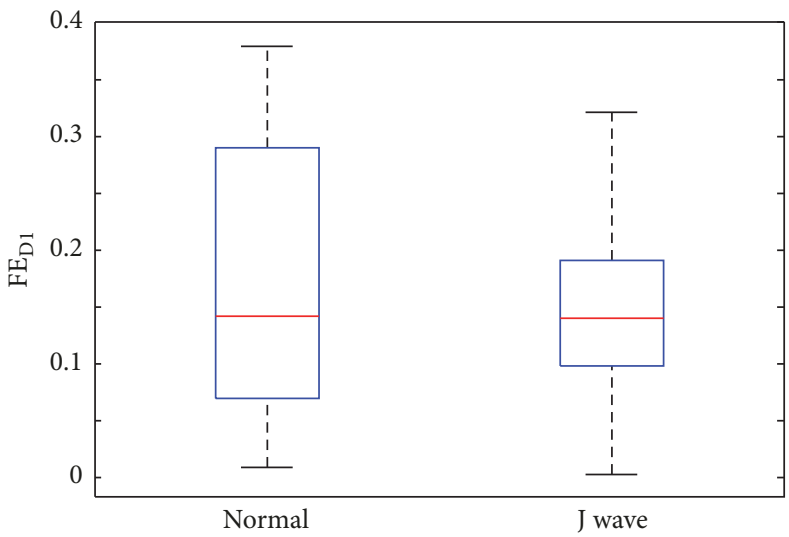

(a)

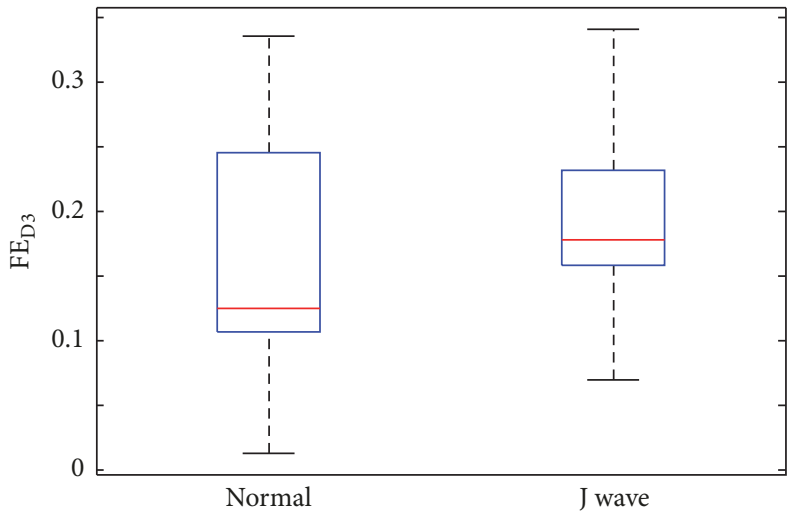

(c)

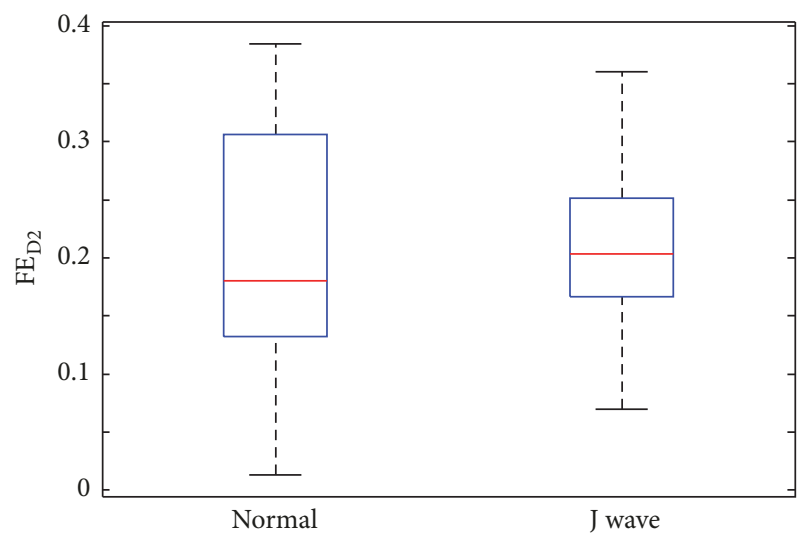

(b)

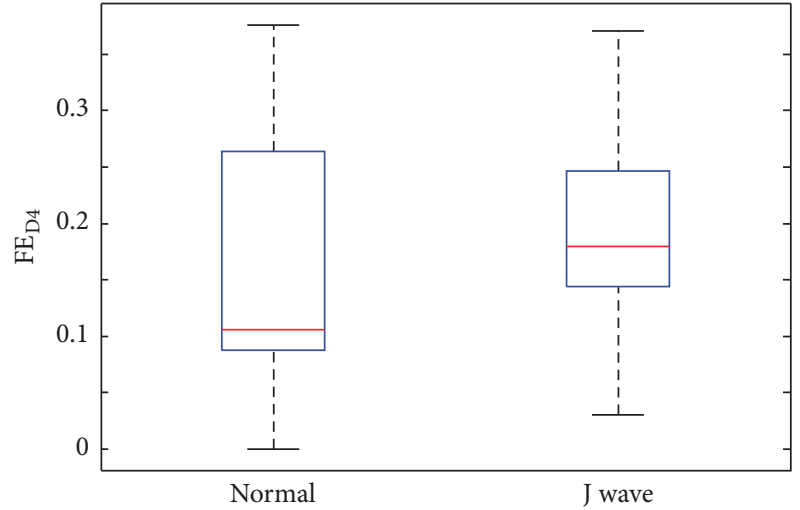

(d)

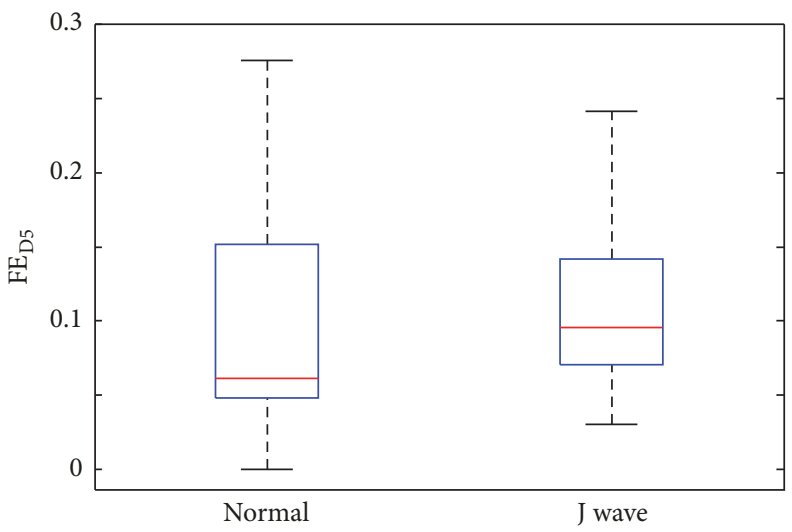

(e)

Figure 3: Boxplots for features at each decomposition level of normal ECG and J wave: (a) Level 1, (b) Level 2, (c) Level 3, (d) Level 4, and (e) Level 5.

TABLE 4: Classification performance of LS-SVM classifier using different kernel functions.

\begin{tabular}{lcccc}
\hline Feature number & Kernel function & ACC (\%) & SEN (\%) & SPE (\%) \\
\hline \multirow{2}{*}{ All features } & RBF & 96.73 & 95.18 & 95.17 \\
& MW & 96.79 & 95.47 & 95.29 \\
Feature selection & RBF & 97.56 & 95.69 & 95.78 \\
& MW & 97.61 & 97.76 & 95.82 \\
\hline
\end{tabular}




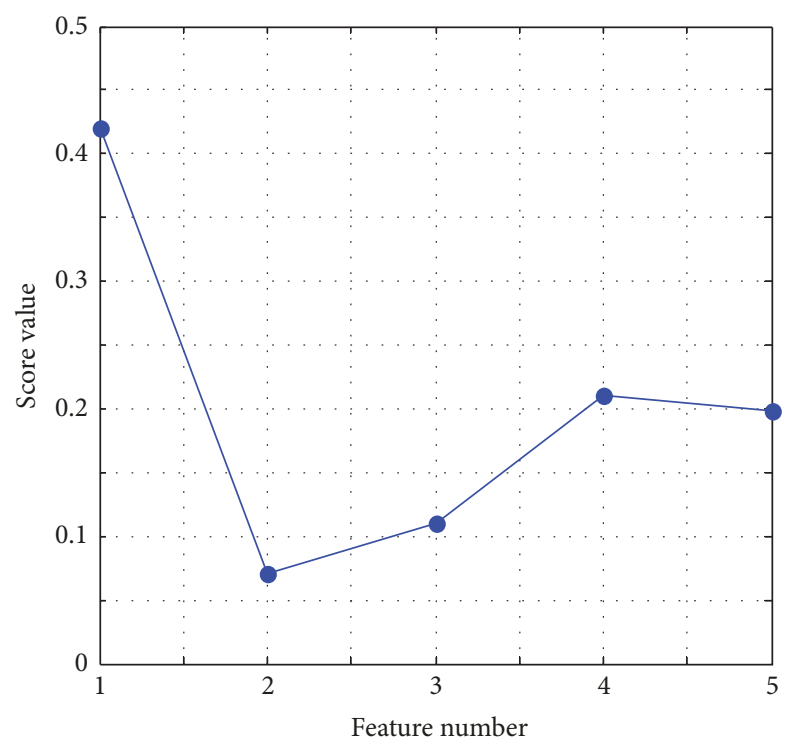

FIGURE 4: Score value of each feature using feature scoring.

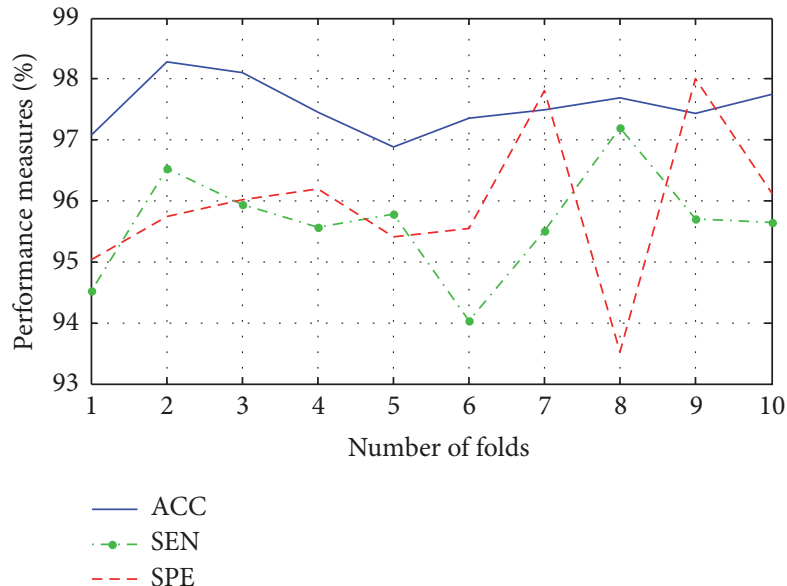

(a) $\mathrm{RBF}$

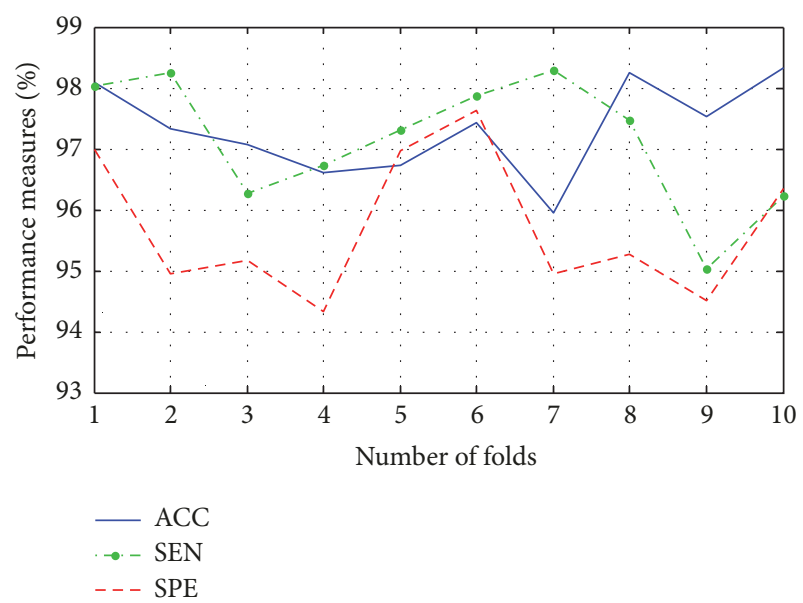

(b) MW

FIGURE 5: Plots showing performance measures versus the number of folds for LS-SVM classifier for kernel function: (a) RBF; (b) MW.

We can also observe that after using feature selection method the values of average accuracy, average sensitivity, and average specificity are higher compared with using all features. The values of the kernel parameters we selected are $\sigma=0.9$ for RBF kernel and $l=1.1$ and $v_{0}=0.35$ for MW kernel. In order to gain a stable and reliable classification performance of LSSVM, tenfold cross validation technique is applied to avoid the possibility of overfitting of the model $[42,43]$. That is, the dataset of features is divided into 10 subsets randomly and consists of one testing subset and nine training subsets. The performances are averaged after ten iterations. ACC, SEN, and SPE for tenfold cross validation method can be seen in Figures 5(a) and 5(b) for RBF and MW kernels, respectively.

Currently, however, only few research teams have studied the automatic detection and classification of J wave from the perspective of signal processing and machine learning.
In [13], a method for automated detection of $\mathrm{J}$ wave is performed. Their method is based on locating a break point in the descending limb of the terminal QRS. New logic was added to Glasgow ECG analysis program to automate the detection of $\mathrm{J}$ wave. The high sensitivity of $90.5 \%$ and specificity of $96.5 \%$ are achieved using this method. A technique based on digital 12-lead electrocardiogram is proposed for the automated J wave detection in [14]. ECG signals were automatically processed with the GE Marquette 12-SL program 2001 version (GE Marquette, Milwaukee, WI). Thereafter, the functional data analysis techniques were applied to the processed ECG signals. The detection sensitivity and specificity are $89 \%$ and $86 \%$, respectively. The two methods have no parameter of accuracy. In [15], a method for automated J wave detection and characterization based on feature extraction is developed. First, five features including 
TABLE 5: Comparison of proposed method with other existing methods of J wave automatic detection.

\begin{tabular}{|c|c|c|c|c|c|}
\hline Authors & Applied methods & Classification & $\operatorname{ACC}(\%)$ & SEN (\%) & SPE (\%) \\
\hline Clark et al. (2014) & \multicolumn{2}{|c|}{$\begin{array}{l}\text { Glasgow ECG analysis program with new } \\
\text { logic }\end{array}$} & 91.3 & 89.5 & 94.5 \\
\hline Wang et al. (2015) & $\begin{array}{l}\text { GE Marquette 12-SL } \\
\text { program } 2001 \text { version }\end{array}$ & $\begin{array}{c}\text { Functional data } \\
\text { analysis }\end{array}$ & 89.6 & 88.45 & 87.8 \\
\hline Li et al. (2015) & $\begin{array}{l}\text { Time-domain features } \\
\text { and discrete wavelet } \\
\text { transform (DWT) }\end{array}$ & $\begin{array}{l}\text { Hidden Markov } \\
\text { model (HMM) }\end{array}$ & 93.35 & 91.32 & 92.2 \\
\hline Li et al. (2015) & $\begin{array}{l}\text { Curve fitting and } \\
\text { wavelet transform } \\
\text { (WT) }\end{array}$ & SVM & 92.58 & 93.21 & 93.87 \\
\hline Li et al. (2016) & $\begin{array}{l}\text { Time-domain } \\
\text { features, power } \\
\text { spectrum, and } \\
\text { cumulative } \\
\text { probability }\end{array}$ & Decision tree (DT) & 96.03 & 95.1 & 96.25 \\
\hline In the present work & $\begin{array}{c}\text { ATFFWT and Fuzzy } \\
\text { Entropy }\end{array}$ & LS-SVM & 97.61 & 97.76 & 95.82 \\
\hline
\end{tabular}

three time-domain features and two wavelet-based features are defined. Then, PCA is used to reduce the dimension of these features. Highest classification accuracy of $92.5 \%$ is attained using HMM. An approach for J wave autodetection is based on support vector machine [16]. CF and WT are used for feature extraction from ECG segments data. Also 92.5\% classification accuracy is reported using SVM classifier. In [17], a novel J wave detection method based on massive ECG data and MapReduce is presented. The power spectrum and the cumulative probability of ECG signals are computed as features. Further, DT is applied to classify and recognize ECG signals. Lastly, they implemented the above process under the parallel programming model MapReduce to handle the massive ECG data and achieved an accuracy of $96.23 \%$.

In the present work, we have developed a novel methodology for automatic detection of J wave. ATFFWT is applied to decompose the processed ECG signals into the desired subbands to capture the hidden useful information from ECG segment beats of 100 normal and $15 \mathrm{~J}$ wave subjects. We have used ATFFWT-based decomposition due to its desirable property; it is able to extract more meaningful information and is suitable for biomedical signals analysis. Furthermore, FE is computed on the decomposed subbands to fetch the information from detail coefficients at each level. FE can measure the similarity based on exponential function in the time series [25]. Feature scoring method is employed for selection, thus removing irrelevant and redundant features, and searching for the optimal feature subset. Finally, these clinically significant features are fed to a LS-SVM classifier with different kernel functions. We have observed an accuracy of $97.56 \%$ and $97.61 \%$ using RBF and MW kernel functions, respectively. Finally, to verify the effectiveness of our method, we have evaluated all methods with the latest collected data, and the summary of performance of other existing methods of J wave automatic detection is shown in Table 5.

\section{Conclusion}

An automated detection of J wave from ECG signals with high speed and accuracy is a great challenge task. In this study, a new technique is proposed to detect the J wave automatically using ECG segment beats. ATFFWT decomposition method and FE extraction are employed to catch the hidden information from ECG signals. Feature scoring method is used to optimize the classification performance. Highest classification performance is founded using LS-SVM classifier with tenfold cross validation procedure while training and testing. The limitation of this work is small dataset; we have used only $15 \mathrm{~J}$ wave subjects. Before the developed effective algorithm can be used to design an expert system to aid clinicians in their regular diagnosis, it needs to be tested using large dataset. And another limitation is that utilization of a fixed beat length is not always optimum because of fast and slow varying heart rhythms. Better methods of adaptive beat size segmentation are needed to study. In the future, the work could be extended in three aspects: (1) it would be of interest to develop an expert model for filter parameters $p, q, r, s$, and $\beta$ optimization of ATTFWT; (2) it is highly desirable that the large dataset would be used to evaluate the proposed technique; (3) the method can be used for other biosignals application, such as electroencephalograph (EEG) and electromyogram (EMG).

\section{Conflicts of Interest}

The authors declare that there are no conflicts of interest regarding the publication of this article.

\section{Acknowledgments}

This work was supported by the National High Technology Research and Development Program (863 Program ) of China 
[Grant no. 2015AA016901, High Linearity Laser Diode Array and High Saturation Power Photodiode Array]; the National Natural Science Foundation of China [Grant no. 61371062, Research on the Theory and Key Technology of J Wave Extraction in ECG Signal]; and the International Cooperation Project of Shanxi Province [Grant no. 201603D421014, Three-Dimensional Reconstruction Research of Quantitative Multiple Sclerosis Demyelination].

\section{References}

[1] G. Roth A, C. Johnson, A. Abajobir et al., "Global, regional, and national burden of cardiovascular diseases for 10 causes, 1990 to 2015," Journal of the American College of Cardiology, 2017.

[2] N. D. Wong, "Epidemiological studies of CHD and the evolution of preventive cardiology," Nature Reviews Cardiology, vol. 11, no. 5, pp. 276-289, 2014.

[3] M. J. Junttila, S. J. Sager, J. T. Tikkanen, O. Anttonen, H. V. Huikuri, and R. J. Myerburg, "Clinical significance of variants of J-points and J-waves: early repolarization patterns and risk," European Heart Journal, vol. 33, no. 21, pp. 2639-2643, 2012.

[4] C. Antzelevitch and G.-X. Yan, "J wave syndromes," Heart Rhythm, vol. 7, no. 4, pp. 549-558, 2010.

[5] M. Badri, A. Patel, and G.-X. Yan, "Cellular and ionic basis of J-wave syndromes," Trends in Cardiovascular Medicine, vol. 25, no. 1, pp. 12-21, 2015.

[6] H. V. Huikuri, "Separation of Benign from Malignant J waves," Heart Rhythm, vol. 12, no. 2, pp. 384-385, 2015.

[7] Y. Aizawa, M. Sato, H. Kitazawa et al., "Tachycardia-dependent augmentation of "notched J waves" in a general patient population without ventricular fibrillation or cardiac arrest: not a repolarization but a depolarization abnormality?" Heart Rhythm, vol. 12, no. 2, pp. 376-383, 2015.

[8] R. J. Martis, U. R. Acharya, and L. C. Min, "ECG beat classification using PCA, LDA, ICA and discrete wavelet transform," Biomedical Signal Processing and Control, vol. 8, no. 5, pp. 437448, 2013.

[9] M. Kumar, R. B. Pachori, and U. R. Acharya, "An efficient automated technique for CAD diagnosis using flexible analytic wavelet transform and entropy features extracted from HRV signals," Expert Systems with Applications, vol. 63, pp. 165-172, 2016.

[10] I. Daubechies, "The wavelet transform, time-frequency localization and signal analysis," IEEE Transactions on Information Theory, vol. 36, no. 5, pp. 961-1005, 1990.

[11] I. Bayram, "An analytic wavelet transform with a flexible timefrequency covering," IEEE Transactions on Signal Processing, vol. 61, no. 5, pp. 1131-1142, 2013.

[12] A. L. Goldberger, L. A. N. Amaral, L. Glass et al., "PhysioBank, PhysioToolkit, and PhysioNet: components of a new research resource for complex physiologic signals [електроннийресурс]," Circulation, vol. 101, pp. e215-e220, 2000.

[13] E. N. Clark, I. Katibi, and P. W. Macfarlane, "Automatic detection of end QRS notching or slurring," Journal of Electrocardiology, vol. 47, no. 2, pp. 151-154, 2014.

[14] Y. Wang, H.-T. Wu, I. Daubechies, Y. Li, E. H. Estes, and E. Z. Soliman, "Automated J wave detection from digital 12-lead electrocardiogram," Journal of Electrocardiology, vol. 48, no. 1, pp. 21-28, 2015.
[15] D. Li, Y. Bai, and J. Zhao, "A method for automated J wave detection and characterisation based on feature extraction," in Big Data Computing and Communications, pp. 421-433, Springer International Publishing, 2015.

[16] D. Li, X. Liu, and J. Zhao, "An approach for J wave autodetection based on support vector machine," in Big Data Computing and Communications, pp. 453-461, Springer International Publishing, 2015.

[17] D. Li, W. Ma, and J. Zhao, "A novel J wave detection method based on massive ECG data and MapReduce," in International Conference on Big Data Computing and Communications, pp. 399-408, Springer International Publishing, 2016.

[18] N. Iyengar, C.-K. Peng, R. Morin, A. L. Goldberger, and L. A. Lipsitz, "Age-related alterations in the fractal scaling of cardiac interbeat interval dynamics," American Journal of PhysiologyRegulatory, Integrative and Comparative Physiology, vol. 271, no. 4, pp. R1078-R1084, 1996.

[19] J. Pan and W. J. Tompkins, "A real-time QRS detection algorithm," IEEE Transactions on Biomedical Engineering, vol. 32, no. 3, pp. 230-236, 1985.

[20] U. R. Acharya, V. K. Sudarshan, J. E. W. Koh et al., "Application of higher-order spectra for the characterization of Coronary artery disease using electrocardiogram signals," Biomedical Signal Processing and Control, vol. 31, pp. 31-43, 2017.

[21] C. L. Zhang, B. Li, B. Q. Chen et al., "Weak fault signature extraction of rotating machinery using flexible analytic wavelet transform," Mechanical Systems and Signal Processing, vol. 64, pp. 162-187, 2015.

[22] M. Kumar, R. B. Pachori, and U. R. Acharya, "Characterization of coronary artery disease using flexible analytic wavelet transform applied on ECG signals," Biomedical Signal Processing and Control, vol. 31, pp. 301-308, 2017.

[23] U. R. Acharya, H. Fujita, M. Adam et al., "Automated characterization and classification of coronary artery disease and myocardial infarction by decomposition of ECG signals: a comparative study," Information Sciences, vol. 377, pp. 17-29, 2017.

[24] M. Kumar, R. B. Pachori, and U. R. Acharya, "Use of accumulated entropies for automated detection of congestive heart failure in flexible analytic wavelet transform framework based on short-term HRV signals," Entropy, vol. 19, no. 3, article 92, 2017.

[25] W. T. Chen, Z. Z. Wang, H. B. Xie, and W. Yu, "Characterization of surface EMG signal based on fuzzy entropy," IEEE Transactions on Neural Systems and Rehabilitation Engineering, vol. 15, no. 2, pp. 266-272, 2007.

[26] R. Sharma and R. B. Pachori, "Classification of epileptic seizures in EEG signals based on phase space representation of intrinsic mode functions," Expert Systems with Applications, vol. 42, no. 3, pp. 1106-1117, 2015.

[27] U. R. Acharya, H. Fujita, V. K. Sudarshan, S. Bhat, and J. E. W. Koh, "Application of entropies for automated diagnosis of epilepsy using EEG signals: a review," Knowledge-Based Systems, vol. 88, pp. 85-96, 2015.

[28] J. Pohjalainen, O. Räsänen, and S. Kadioglu, "Feature selection methods and their combinations in high-dimensional classification of speaker likability, intelligibility and personality traits," Computer Speech and Language, vol. 29, no. 1, pp. 145-171, 2015.

[29] C. Camara, K. Warwick, R. Bruña, T. Aziz, F. del Pozo, and F. Maestú, "A fuzzy inference system for closed-loop deep brain stimulation in Parkinson's disease," Journal of Medical Systems, vol. 39, no. 11, article 155, 2015. 
[30] S. Aydın, M. A. Tunga, and S. Yetkin, "Mutual information analysis of sleep EEG in detecting psycho-physiological insomnia," Journal of Medical Systems, vol. 39, no. 5, p. 43, 2015.

[31] J. A. K. Suykens and J. Vandewalle, "Least squares support vector machine classifiers," Neural Processing Letters, vol. 9, no. 3, pp. 293-300, 1999.

[32] A. H. Khandoker, D. T. H. Lai, R. K. Begg, and M. Palaniswami, "Wavelet-based feature extraction for support vector machines for screening balance impairments in the elderly," IEEE Transactions on Neural Systems and Rehabilitation Engineering, vol. 15, no. 4, pp. 587-597, 2007.

[33] V. Bajaj and R. B. Pachori, "Classification of seizure and nonseizure EEG signals using empirical mode decomposition," IEEE Transactions on Information Technology in Biomedicine, vol. 16, no. 6, pp. 1135-1142, 2012.

[34] M. Zavar, S. Rahati, M.-R. Akbarzadeh-T, and H. Ghasemifard, "Evolutionary model selection in a wavelet-based support vector machine for automated seizure detection," Expert Systems with Applications, vol. 38, no. 9, pp. 10751-10758, 2011.

[35] R. B. Pachori, M. Kumar, and P. Avinash, "An improved online paradigm for screening of diabetic patients using RR-interval signals," Journal of Mechanics in Medicine and Biology, vol. 16, no. 01, Article ID 1640003, 2016.

[36] S. Patidar and R. B. Pachori, "Classification of cardiac sound signals using constrained tunable-Q wavelet transform," Expert Systems with Applications, vol. 41, no. 16, pp. 7161-7170, 2014.

[37] R. Sharma, R. B. Pachori, and U. Rajendra Acharya, "An integrated index for the identification of focal electroencephalogram signals using discrete wavelet transform and entropy measures," Entropy, vol. 17, no. 8, pp. 5218-5240, 2015.

[38] R. Sharma, R. B. Pachori, and U. R. Acharya, "Application of entropy measures on intrinsic mode functions for the automated identification of focal electroencephalogram signals," Entropy, vol. 17, no. 2, pp. 669-691, 2015.

[39] M. Sharma, R. B. Pachori, and U. Rajendra Acharya, "A new approach to characterize epileptic seizures using analytic timefrequency flexible wavelet transform and fractal dimension," Pattern Recognition Letters, vol. 94, pp. 172-179, 2017.

[40] S. Maheshwari, R. B. Pachori, and U. R. Acharya, "Automated diagnosis of glaucoma using empirical wavelet transform and correntropy features extracted from fundus images," IEEE Journal of Biomedical and Health Informatics, vol. 21, no. 3, pp. 803-813, 2017.

[41] P. E. McKight and J. Najab, "Kruskal-Wallis test," Corsini Encyclopedia of Psychology, 2010.

[42] S.-N. Yu and Y.-H. Chen, "Electrocardiogram beat classification based on wavelet transformation and probabilistic neural network," Pattern Recognition Letters, vol. 28, no. 10, pp. 1142-1150, 2007.

[43] R. Kohavi, "A study of cross-validation and bootstrap for accuracy estimation and model selection," International Joint Conferences on Artificial Intelligence, vol. 14, no. 2, pp. 1137-1145, 1995. 


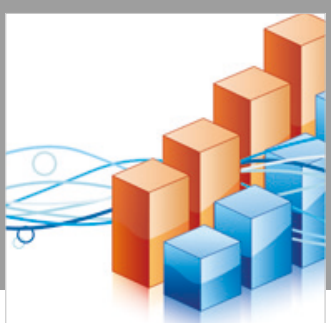

Advances in

Operations Research

\section{-n-m}
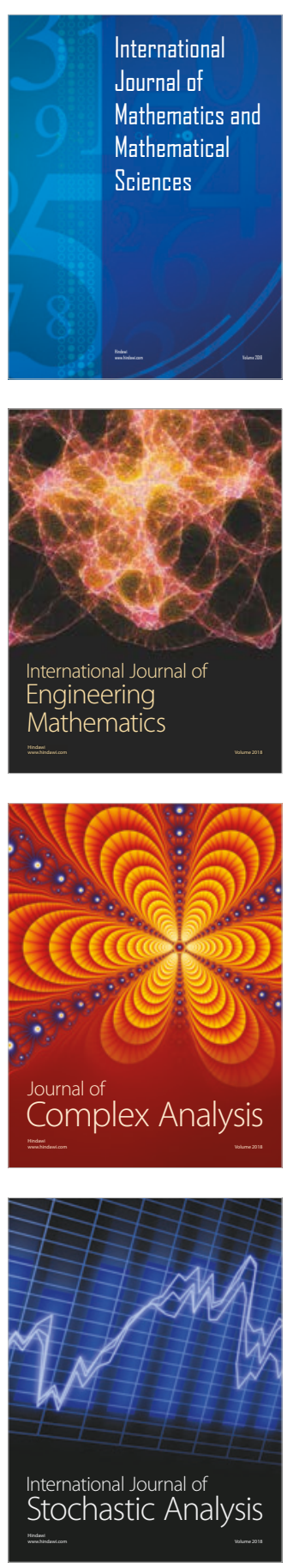
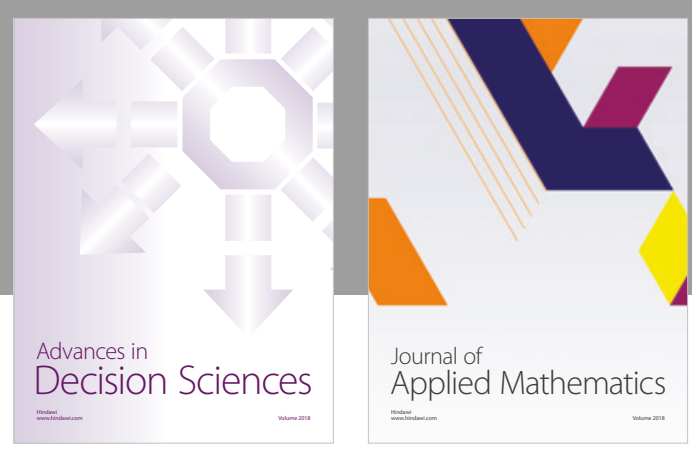

Journal of

Applied Mathematics
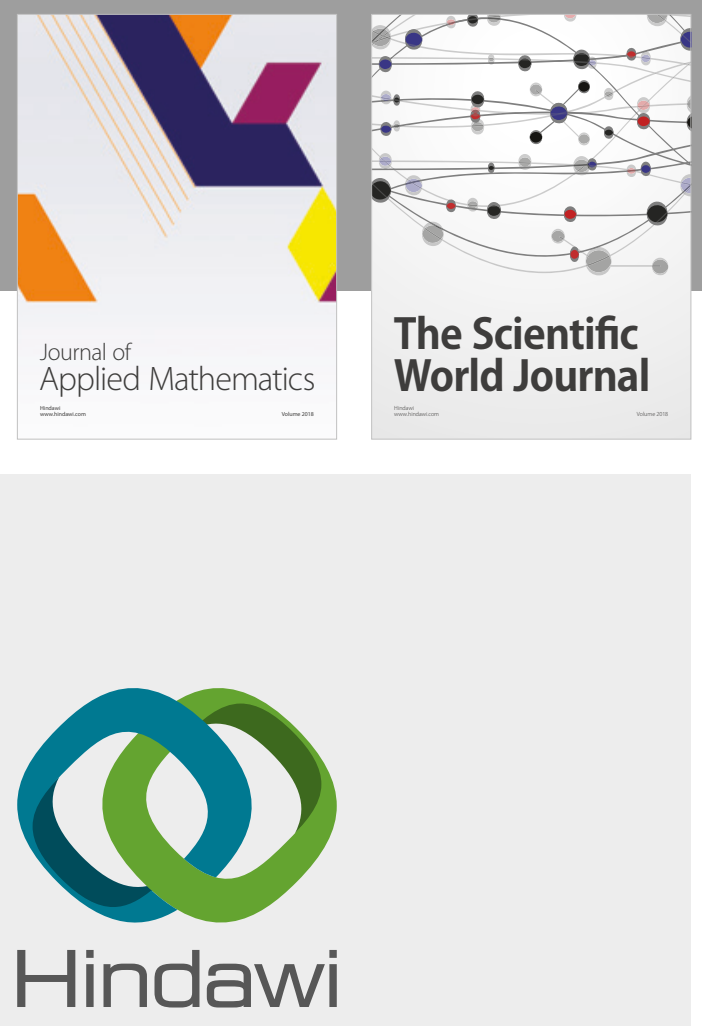

Submit your manuscripts at

www.hindawi.com

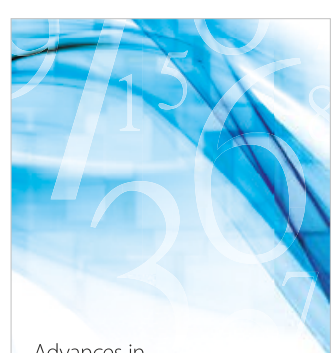

Advances in
Numerical Analysis
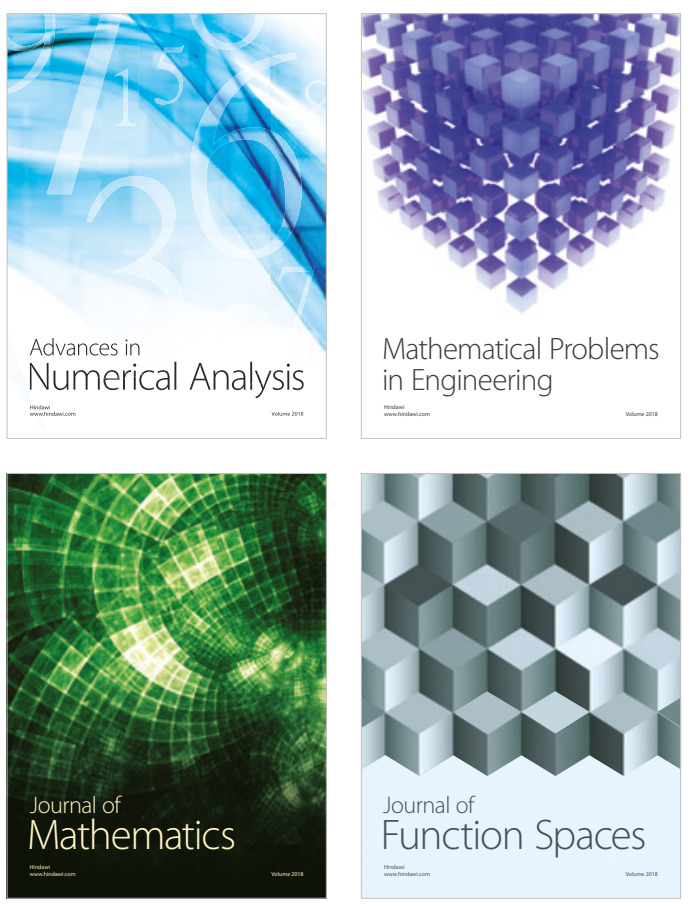

Mathematical Problems in Engineering

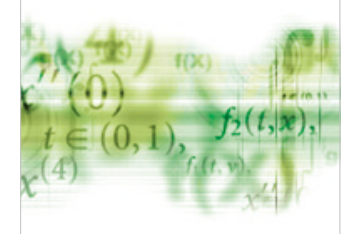

International Journal of

Differential Equations

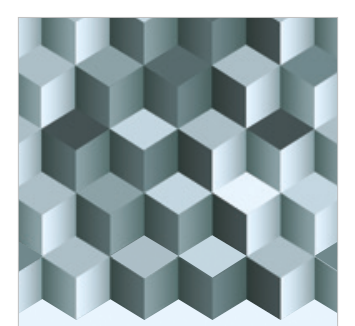

Journal of

Function Spaces

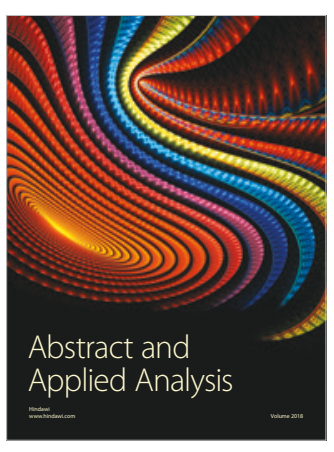

The Scientific

World Journal

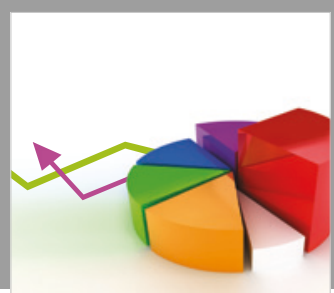

Journal of

Probability and Statistics
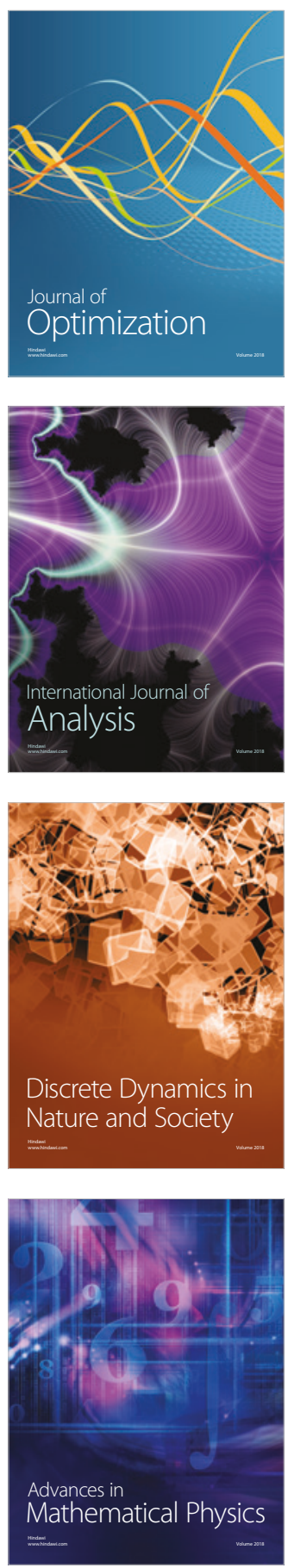